https://helda.helsinki.fi

\title{
Listeria monocytogenes contamination pattern in pig slaughterhouses
}

\author{
Autio, T. \\ International Association for Food Protection \\ 2000
}

Journal of food protection. 2000. 63. 1438-1442

http://hdl.handle.net/1975/765

Downloaded from Helda, University of Helsinki institutional repository.

This is an electronic reprint of the original article.

This reprint may differ from the original in pagination and typographic detail.

Please cite the original version. 


\title{
Listeria monocytogenes Contamination Pattern in Pig Slaughterhouses
}

\author{
T. AUTIO,* T. SÄTERI, M. FREDRIKSSON-AHOMAA, M. RAHKIO, J. LUNDÉN, AND H. KORKEALA \\ Department of Food and Environmental Hygiene, Faculty of Veterinary Medicine, University of Helsinki, Finland
}

MS 00-23: Received 25 January 2000/Accepted 2 June 2000

\begin{abstract}
Ten low-capacity slaughterhouses were examined for Listeria by collecting a total of 373 samples, of which 50, 250, and 73 were taken from carcasses, pluck sets, and the slaughterhouse environment, respectively. Six slaughterhouses and $9 \%$ of all samples were positive for Listeria monocytogenes. Of the samples taken from pluck sets, $9 \%$ were positive for $L$. monocytogenes, the highest prevalence occurring in tongue and tonsil samples, at $14 \%$ and $12 \%$, respectively. Six of $50(12 \%)$ carcasses were contaminated with $L$. monocytogenes. In the slaughterhouse environment, $L$. monocytogenes was detected in two, one, one, and one sample originating from the saws, drain, door, and table, respectively. Carcasses were contaminated with $L$. monocytogenes in those two slaughterhouses, where the mechanical saws, used for both brisket and back splitting, were also positive for $L$. monocytogenes. A total of $58 \mathrm{~L}$. monocytogenes isolates were characterized by pulsed-field gel electrophoresis typing. The isolates were divided into 18 pulsotypes, 15 of which were detected in pluck sets. In two slaughterhouses, where the carcasses were contaminated with $L$. monocytogenes, the same pulsotypes were also recovered from splitting saws. In addition, identical pulsotypes were recovered from pluck sets. Our findings indicate that $L$. monocytogenes of tongue and tonsil origin may contaminate the slaughtering equipment that may in turn spread the pathogen to carcasses. Thus, it is of the utmost importance to follow good manufacturing practices and to have efficient cleaning and disinfection procedures to prevent equipment being contaminated with $L$. monocytogenes.
\end{abstract}

Listeria monocytogenes has been the causative agent of several foodborne listeriosis outbreaks involving milk and dairy products, vegetables, salads, meat products, and fishery products $(10)$. Thus, the prevention of $L$. monocytogenes contamination in food products is of major importance. In order to be able to prevent contamination of food items by the bacterium, the contamination routes and the initial sources of $L$. monocytogenes contamination must be identified.

Listeria has been recovered from slaughterhouse environments, and the carcasses, tonsils, and feces of animals $(6,11,14,18,19,21)$. It has been suggested that Listeria detected in carcasses may have a nonfecal origin and that a possible contamination source may be the equipment (17). However, the initial source and contamination sites of Listeria during pig slaughtering remain obscure. To better understand Listeria contamination, it is important to determine the similarities and dissimilarities of isolates recovered from different origins. Genotypic typing methods have been used to track contaminants in food-processing facilities (2, $8,16)$. By comparing the isolates of different origins, it is possible to trace the contamination routes and establish the initial source of plant contamination.

This study was set up to establish the prevalence and possible contamination routes of $L$. monocytogenes in pig

* Author for correspondence. Tel: +358-9-191 49 766; Fax: +358-9-191

49 718; E-mail: Tiina.Jo.Autio@Helsinki.fi. slaughterhouses. Contamination sites were identified by sampling the environment, pluck sets, and carcasses of 10 low-capacity slaughterhouses and by typing isolates using pulsed-field gel electrophoresis (PFGE) $(4,5,25)$, which is regarded as the gold standard for typing.

\section{MATERIALS AND METHODS}

Sampling. In low-capacity slaughterhouses, the maximum amount of slaughtering is 20 animal units/week and 1,000 animal units/year (9). Pigs' body weights of $>100 \mathrm{~kg}$ are counted as 0.2 and other as 0.15 animal units. There are 57 low-capacity pig slaughterhouses in Finland. Ten geographically representative slaughterhouses, having no apparent association with each other, were examined for Listeria spp. A total of 373 samples were collected, of which 50, 250, and 73 samples were from carcasses, pluck sets, and slaughterhouse environment, respectively. The carcasses and pluck sets (tongue, tonsil, heart, liver, kidney) were sampled by swabbing with $7.5-\mathrm{cm}$ by $7.5-\mathrm{cm}$ sterile gauze-pads premoistened in sterile $0.1 \%$ peptone water. The backs of carcasses were sampled after splitting (Fig. 1), and pluck sets were sampled by swabbing the entire surface of offals hanging from hooks. The slaughterhouse environment, equipment, and employee aprons were sampled by sponge (Technical Service Consultants Ltd., Heywood, Lancashire, UK) sampling technique $(7,20)$, and drains were examined by collecting $25 \mathrm{ml}$ of drain water. The environmental samples in slaughterhouses $2,4,5,7$, and 8 were obtained prior to slaughtering and in slaughterhouses 1, 3, 6, 9, and 10 after slaughtering. The slaughterhouses to be sampled before of after slaughtering were chosen at random. 


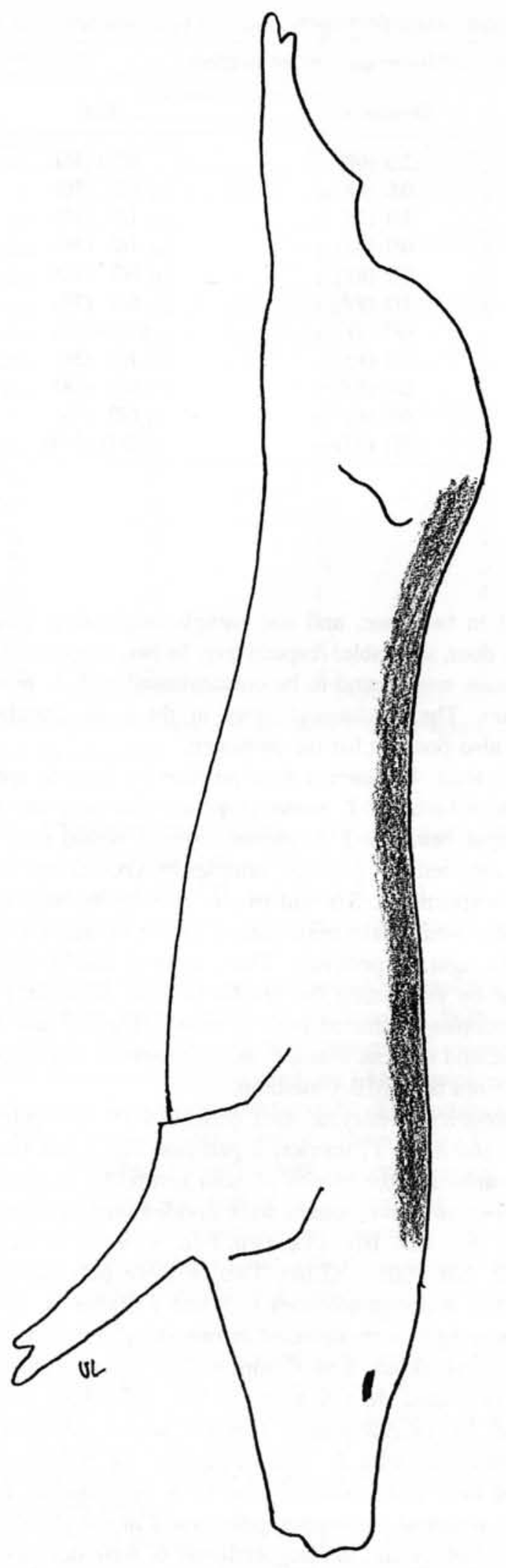

FIGURE 1. Sampled areas on pork carcasses (indicated in black).

Bacteriological analysis. Examination for L. monocytogenes was carried out using a one-step enrichment method according to the Nordic Committee on Food Analysis (1), with the addition of LMBA agar plate (13) (trypticase soy agar base [Difco, Detroit, Mich.], $10 \mathrm{~g} /$ liter lithium chloride, $10 \mathrm{mg} /$ liter polymyxine B sulfate [Sigma Chemicals, St. Louis, Mo.], $20 \mathrm{mg} /$ liter ceftazidime
TABLE 1. Prevalence of Listeria spp. and L. monocytogenes in pluck sets, carcasses and environment of 10 slaughterhouses

\begin{tabular}{lccc}
\hline & \multicolumn{2}{c}{$\begin{array}{c}\text { No. of positive } \\
\text { samples }(\%)\end{array}$} \\
\cline { 3 - 4 } \multicolumn{1}{c}{ Source } & $\begin{array}{c}\text { No. of } \\
\text { samples }\end{array}$ & $\begin{array}{c}\text { Listeria } \\
\text { spp. }{ }^{a}\end{array}$ & $\begin{array}{c}\text { L. mono- } \\
\text { cytogenes }\end{array}$ \\
\hline Pluck sets & & & \\
Tongues & 50 & $8(16)$ & $7(14)$ \\
Tonsils & 50 & $6(12)$ & $6(12)$ \\
Hearts & 50 & $3(6)$ & $3(6)$ \\
Livers & 50 & $3(6)$ & $3(6)$ \\
Kidneys & 50 & $4(8)$ & $3(6)$ \\
Carcasses & 50 & $6(12)$ & $6(12)$ \\
Environment & & & $1(10)$ \\
Drains & 10 & $4(40)$ & 0 \\
Aprons & 10 & $1(10)$ & $1(10)$ \\
Tables & 10 & $1(10)$ & 0 \\
Knives & 10 & 0 & $1(10)$ \\
Doors & 10 & $1(10)$ & $2(20)$ \\
Saws & 10 & $3(30)$ & 0 \\
Others & 13 & $1(10)$ & $33(9)$ \\
Total & 373 & $41(11)$ & \\
\hline
\end{tabular}

${ }^{a}$ Includes L. monocytogenes.

[Abtek Biologicals Ltd, Liverpool, England], 5\% sterile defibrinated sheep blood). Each sample was incubated in Listeria enrichment broth (Oxoid, Basingstoke, UK) at $30^{\circ} \mathrm{C}$ for $48 \mathrm{~h}$. Enrichment broth was plated on Oxford agar (Oxoid) and on LMBA agar plates. The plates were incubated at $37^{\circ} \mathrm{C}$ for $48 \mathrm{~h}$. Five suspected colonies from both Oxford agar and LMBA agar were streaked on blood agar. The $\beta$-hemolytic colonies were identified by Gram staining, catalase reaction, motility microscopically at $25^{\circ} \mathrm{C}$, and further identified using API Listeria (BioMériux SA, Marcy l'Etoile, France). When no $\beta$-hemolytic colonies were observed, the nonhemolytic colonies were further identified as described above. Two L. monocytogenes isolates from each positive sample, one from each selective agar, were maintained frozen in Protect bacterial preservation tubes (Technical Service Consultants) at $-70^{\circ} \mathrm{C}$ prior to serotyping and PFGE typing.

In situ DNA isolation and PFGE. A total of $58 \mathrm{~L}$. monocytogenes isolates were characterized by PFGE. In situ DNA isolation and PFGE were performed as described by Autio et al. (2). ApaI and AscI (New England Biolabs, Beverly, Mass.) were used for restriction endonuclease digestion. The samples were electrophoresed as described by Autio et al. (2). The pulse times for ApaI and AscI ramped from $1 \mathrm{~s}$ to $35 \mathrm{~s}$ for $18 \mathrm{~h}$. Low range PFG markers (New England Biolabs) were used for fragment size determination. The gels were stained with ethidium bromide and were digitally photographed with an Alpha Imager 2000 documentation system (Alpha Innotech, San Leandro, Calif.). The genotypes were considered closely related when a three-fragment difference was not exceeded (23).

Serotyping. One strain from each pulsotype was serotyped with commercial antisera (Denka Seiken, Tokyo, Japan) according to manufacturer instructions.

\section{RESULTS}

Listeria spp. was detected in $11 \%$ of samples (Table 1). Listeria species recovered were L. monocytogenes and 
TABLE 2. Prevalence of Listeria spp. and L. monocytogenes in 10 low-capacity slaughterhouses

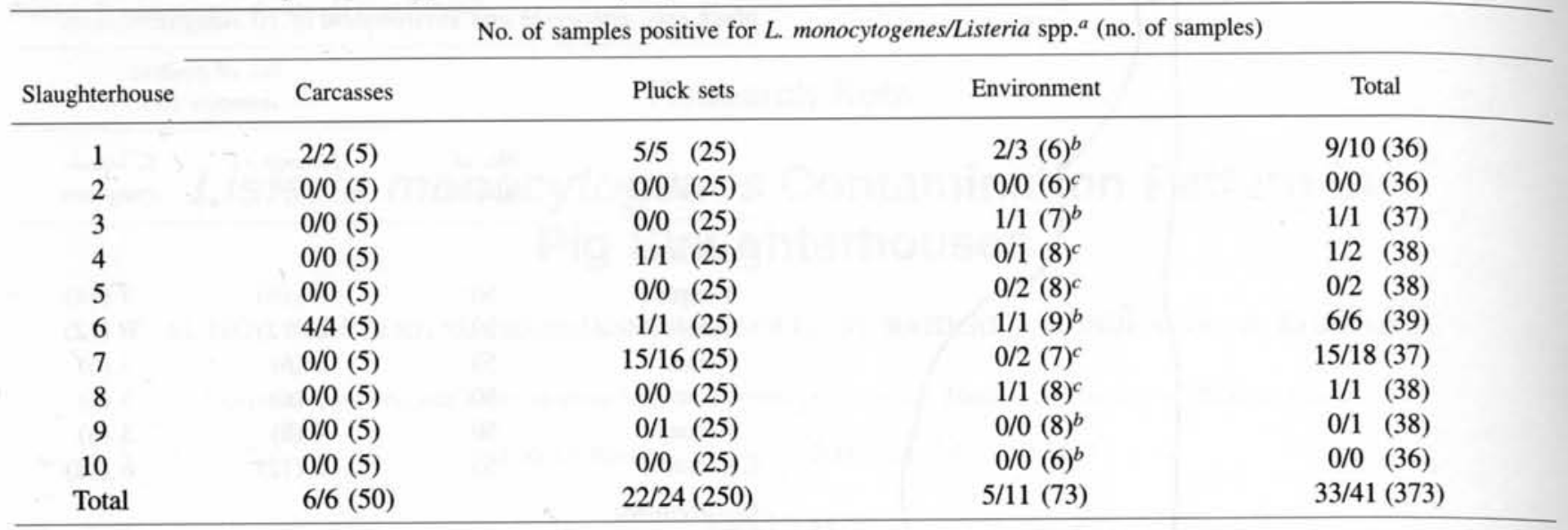

${ }^{a}$ Includes L. monocytogenes.

${ }^{b}$ Samples taken after slaughtering.

c Samples taken prior to slaughtering.

Listeria innocua. L. monocytogenes was detected in $9 \%$ of samples. Altogether $10 \%$ of pluck set samples were positive for Listeria spp. and $9 \%$ for L. monocytogenes. The highest prevalence of $L$. monocytogenes was detected in tongue and tonsil samples, at $14 \%$ and $12 \%$, respectively, whereas contamination of hearts, kidneys, and livers was $6 \%$. Six of 50 (12\%) carcasses were contaminated with $L$. monocytogenes.

Listeria was detected in 8 of 10 slaughterhouses (Table 2). The incidence of Listeria spp. and L. monocytogenes varied from 0 to $49 \%$ and from 0 to $40 \%$, respectively. In five slaughterhouses, Listeria spp. was detected in pluck sets, with slaughterhouse 7 having a particularly high level $(64 \%)$ of contamination.

In the slaughterhouse environment, Listeria spp. and $L$. monocytogenes were detected in $15 \%$ and $7 \%$ of samples, respectively. $L$. monocytogenes was detected in one of five slaughterhouses where environmental samples had been collected prior to slaughtering. The sampling site was the drain. In slaughterhouses where environmental samples were taken after slaughtering, L. monocytogenes was de-

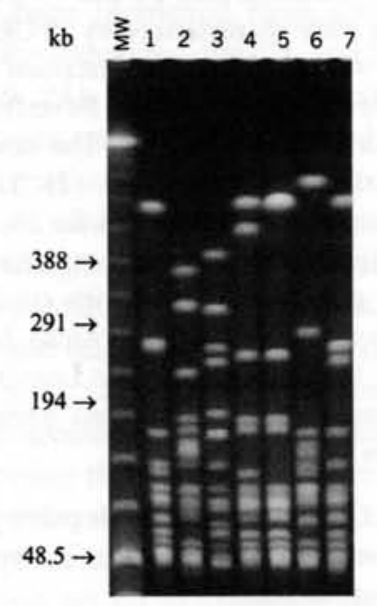

FIGURE 2. Apal digests of L. monocytogenes strains. Lanes: $M W$, low range PFGE marker; 1 , pulsotype VI; 2, pulsotype XII; 3 , pulsotype X; 4, pulsotype XIIIa; 5, pulsotype XIIIb; 6, pulsotype VII; 7 , pulsotype VIII. tected in two, one, and one sample originating from the saws, door, and table, respectively. In two slaughterhouses, carcasses were found to be contaminated with $L$. monocytogenes. The mechanical saws in these slaughterhouses were also positive for the pathogen.

In total, 41 samples were positive for Listeria spp., 33 of which harbored L. monocytogenes. Had only one selective agar been used, L. monocytogenes would only have been detected in 27 and 31 samples by Oxford and LMBA agar, respectively. Six and two $L$. monocytogenes-positive samples would have been missed by use of only Oxford or LMBA agar, respectively. Thus, optimal results were obtained by combining the results of both selective media. Six samples contained both $L$. monocytogenes and $L$. innocua, and in these cases, $L$. monocytogenes was recovered only from the LMBA medium.

Restriction enzyme AscI generated 16 restriction patterns, and ApaI, 17 restriction patterns (Fig. 2 and Table 3). By combining the results of both restriction enzymes, 58 L. monocytogenes isolates were divided into 18 pulsotypes (I, II, IIIa, IIIlb, IIIc, IVa, IVb, IVc, V, VI, VII, VIII, IX, $\mathrm{X}, \mathrm{XI}, \mathrm{XII}, \mathrm{XIII}, \mathrm{XIIIb})$. Two or more pulsotypes were detected in slaughterhouses 1,3 , and 7 (Table 4). At most, 10 pulsotypes were detected in one slaughterhouse, and in a single pluck set, a maximum of five different pulsotypes was recovered. In five samples, two pulsotypes were detected. In slaughterhouses 1 and 6 , where carcasses were contaminated with $L$. monocytogenes, the pulsotypes detected were also recovered from saws. Furthermore, in both cases, identical pulsotypes, pulsotype I in slaughterhouse 1 and pulsotype IIIc in slaughterhouse 6 , were detected from pluck sets. One strain from each pulsotype was serotyped. The strains from pulsotypes II, IIIa, IIIb, IIIc, IVa, IVb, IVc, V, VI, VII, VIII, IX, X, XI, XII, XIIIa, XIIIb were serotype $1 / 2 \mathrm{a}$, and from pulsotype I, serotype $1 / 2 \mathrm{c}$.

\section{DISCUSSION}

Eight slaughterhouses were contaminated with Listeria, with only two slaughterhouses being Listeria negative. This 
TABLE 3. Pulsotypes of L. monocytogenes isolates detected in slaughterhouses

\begin{tabular}{cllc}
\hline & \multicolumn{2}{c}{ Restriction pattern $^{a}$} & \\
\cline { 2 - 3 } Pulsotype & AscI & ApaI & No. of isolates \\
\hline I & A1 & A & 8 \\
II & A2 & B & 6 \\
IIIa & B1 & C1 & 2 \\
IIIb & B2 & C4 & 1 \\
IIIc & B2 & C1 & 12 \\
IVa & C1 & C2 & 1 \\
IVb & C1 & C3 & 2 \\
IVc & C2 & C5 & 1 \\
V & D & D & 1 \\
VI & E & E1 & 5 \\
VII & F & F & 1 \\
VIII & G & E2 & 3 \\
IX & H & G & 2 \\
X & I & H & 1 \\
XI & J1 & I & 6 \\
XII & J2 & J & 1 \\
XIIIa & K1 & K1 & 3 \\
XIIIb & K2 & K2 & 2 \\
\hline
\end{tabular}

${ }^{a}$ Restriction patterns share a letter when a three-fragment difference is not exceeded.

result is in agreement with findings in high-capacity slaughterhouses by Gill and Jones (12), Sammarco et al. (19), Saide-Albornoz et al. (18), and Korsak et al. (14), all of whom describe a variable occurrence of Listeria among slaughterhouses.

Differences between selective media in detection of $L$. monocytogenes were present. In six samples, both $L$. innocua and $L$. monocytogenes were detected, $L$. monocytogenes having been recovered from the LMBA medium. On LMBA media, L. monocytogenes can be distinguished from other Listeria spp. by detection of $\beta$-hemolysis. The best result was obtained by using both selective media. This finding is consistent with that of other researchers (26), who recommend use of multiple plating. However, even better results may have been obtained with the combined use of multiple enrichment media and more than one selective plating agar (22).

A total of 18 pulsotypes were identified by PFGE typing. In five samples, two pulsotypes were detected from a single sample. This is in agreement with previous studies $(2,15)$ and stresses the importance of characterization of several isolates from a single sample in epidemiological and contamination studies.

No identical pulsotypes were detected among slaughterhouses. However, closely related strains were recovered from slaughterhouses 1,3 , and 6 , as well as from slaughterhouses 3 and 7, having no apparent association with each other. Carcasses in two slaughterhouses were contaminated with $L$. monocytogenes. In these slaughterhouses, the saws used for splitting the carcasses were also contaminated with the pathogen. Moreover, the isolates from the saws and the carcasses were determined by PFGE typing to have identical restriction patterns. This suggests that the saws may
TABLE 4. Distribution of L. monocytogenes pulsotypes in pig slaughterhouses

\begin{tabular}{crcc}
\hline \multirow{2}{*}{$\begin{array}{c}\text { Slaughter- } \\
\text { house }\end{array}$} & \multicolumn{3}{c}{ Pulsotypes (no. of isolates) } \\
\cline { 2 - 4 } 1 & Pluck sets & Carcasses & Environment \\
\hline & I (2) & I (3) & I (3) \\
& II (6) & & \\
3 & IIIa (2) & & IVa (1) \\
4 & & & IIIb (1) \\
6 & IX (2) & & IIIc (2) \\
7 & IIIc (2) & IIIc (8) & \\
& IVb (2) & & \\
& IVc (1) & & \\
& VI (5) & & \\
& VII (1) & \\
& VIII (3) & & \\
& X (1) & & \\
7 & XI (6) & & \\
& XII (1) & & \\
& XIIIa (3) & & \\
\hline
\end{tabular}

be the contamination site of the carcasses in these slaughterhouses. This result concurs with the study of Nesbakken et al. (17) that proposes slaughterhouse equipment as a possible source of carcass contamination.

However, the initial origin of Listeria contamination has been unclear. Although Listeria may be present in pig feces, Listeria detected in carcasses has been speculated to have a nonfecal origin (18), and that animal strains do not easily contaminate meat and are replaced by other better adapted strains (3). Bunčić (6) showed pigs to be carriers more often of $L$. monocytogenes $(45 \%)$ in the tonsillar tissue than excretors of the bacteria in feces. Unfortunately, Bunčić (6) neither examined the carcasses nor used any typing methods to investigate the role of tonsils as a possible source of carcass contamination. In our study, $14 \%$ of tongues and $12 \%$ of tonsils were found to harbor $L$. monocytogenes. The saws used for splitting carcasses were also used as brisket saws, and therefore, it is possible that the origin of Listeria on the saws was the tongues and tonsils. Moreover, the pulsotypes detected on the saws after slaughtering and on the carcasses were also found in the pluck sets. Fifteen pulsotypes were detected in pluck sets, and at most, five pulsotypes were detected in a single pluck set. Therefore, it is possible that a wide range of $L$. monocytogenes strains initially enter the plant with the animals and only a few of them have characteristics enabling them to survive, adapt to, and colonize in the processing environment. This is supported by earlier findings of dominant $L$. monocytogenes clones in food-processing environments, strains that have proven to be able to persist for several years $(16,24)$.

Pluck sets in slaughterhouse 7 were found to be contaminated heavily with Listeria. In this slaughterhouse, the pluck sets had been rinsed with tap water, which might have increased dissemination of Listeria from the tonsils and 
tongues through the pluck set. However, the pluck sets were also rinsed in slaughterhouse 9 , but only one pluck set sample was Listeria positive. This may be due to differences in the occurence of $L$. monocytogenes carrier pigs.

It is pertinent to ask whether the contamination results we obtained in low-capacity pig slaughterhouses would also be valid in high-capacity slaughterhouses. The prevalence and diversity results of $L$. monocytogenes in pluck sets can be generalized because, in many cases, pigs from the same farm are slaughtered both in low- and high-capacity slaughterhouses. When pig tonsils are removed together with the pluck set (tongue, esophagus, trachea, lungs, heart, diaphragm, kidneys, and liver), Listeria may spread from the tonsils and tongue to the remainder of the pluck set and carcass. Mechanical equipment in abattoirs may become contaminated either directly or indirectly with Listeria originating from tonsils and tongues. Therefore, it is essential to follow good manufacturing practices and to utilize efficient cleaning and disinfection procedures to prevent equipment contamination. In addition, more research must be conducted in high-capacity slaughterhouses to establish the role of tonsils and tongues in Listeria contamination.

\section{ACKNOWLEDGMENTS}

We are grateful to Sirkku Ekström, Suvi Myyrä, and Diego Gabanillas Gonzales for excellent technical assistance. This work was supported by the Walter Ehrström Foundation and the Finnish Veterinary Foundation.

\section{REFERENCES}

1. Anonymous. 1990. Listeria monocytogenes. Detection in foods. Nordic Committee on Food Analysis. No. 136. Espoo, Finland.

2. Autio, T., S. Hielm, M. Miettinen, A.-M. Sjöberg, K. Aarnisalo, J. Björkroth, T. Mattila-Sandholm, and H. Korkeala. 1999. Sources of Listeria monocytogenes contamination in a cold-smoked rainbow trout processing plant detected by pulsed-field gel electrophoresis typing. Appl. Environ. Microbiol. 65:150-155.

3. Boerlin, P., and J.-C. Piffaretti. 1991. Typing of human, animal, food and environmental isolates of Listeria monocytogenes by multilocus enzyme electrophoresis. Appl. Environ. Microbiol. 57:1624-1629.

4. Brosch, R., C. Buchrieser, and J. Rocourt. 1991. Subtyping of Listeria monocytogenes serovar $4 \mathrm{~b}$ by use of low-frequency-cleavage restriction endonucleases and pulsed-field gel electrophoresis. Res. Microbiol. 142:667-675.

5. Buchrieser, C., R. Brosch, B. Catimel, and J. Rocourt. 1993. Pulsedfield gel electrophoresis applied for comparing Listeria monocytogenes strains involved in outbreaks. Can. J. Microbiol. 39:395-401.

6. Bunčić, S. 1991. The incidence of Listeria monocytogenes in slaughtered animals, in meat, and in meat products in Yugoslavia. Int. J. Food Microbiol. 12:173-180.

7. Daley, E. F., F. Pagotto, and J. M. Farber. 1995. The inhibitory properties of various sponges on Listeria spp. Lett. Appl. Microbiol. 20: 195-198.

8. Destro, M. T., M. F. Leitao, and J. M. Farber. 1996. Use of molecular typing methods to trace the dissemination of Listeria monocytogenes in a shrimp processing plant. Appl. Environ. Microbiol. 62:705-711.

9. European Parliament and the Commission of the European Com- munities. 1995. European Parliament and the Commission Directive 95/23/EC. Off. J. Eur. Commun. 243:7-13.

10. Farber, J. M., and P. I. Peterkin. 1991. Listeria monocytogenes, a food-borne pathogen. Microbiol. Rev. 55:476-511.

11. Fenlon, D. R., J. Wilson, and W. Donachie. 1996. The incidence and level of Listeria monocytogenes contamination of food sources at primary production and initial processing. J. Appl. Bacteriol. 81: 641-650.

12. Gill, C. O., and T. Jones. 1995. The presence of Aeromonas, Listeria and Yersinia in carcass processing equipment at two pig slaughtering plants. Food Microbiol. 12:135-141.

13. Johansson, T. 1998. Enhanced detection and enumeration of Listeria monocytogenes from foodstuffs and food-processing environments. Int. J. Food Microbiol. 40:77-85.

14. Korsak, N., G. Daube, Y. Ghafir, A. Chahed, S. Jolly, and H. Vindevogel. 1998. An efficient sampling technique used to detect four foodborne pathogens on pork and beef carcasses in nine Belgian abattoirs. J. Food Prot. 61:535-541.

15. Loncarevic, S., W. Tham, and M. L. Danielsson-Tham. 1996. The clones of Listeria monocytogenes detected in food depend on the method used. Lett. Appl. Microbiol. 22:381-384.

16. Miettinen, M. K., J. Björkroth, and H. Korkeala. 1999. Characterization of Listeria monocytogenes from an ice cream plant by serotyping and pulsed-field gel electrophoresis. Int. J. Food. Microbiol. 46:187-192.

17. Nesbakken, T., E. Nerbrink, O.-J. Røtterud, and E. Borch. 1994. Reduction of Yersinia enterocolitica and Listeria spp. on pig carcasses by enclosure of the rectum during slaughter. Int. J. Food Microbiol. 23:197-208.

18. Saide-Albornoz, J. J., C. L. Knipe, E. A. Murano, and G. W. Beran. 1995. Contamination of pork carcasses during slaughter, fabrication and chilled storage. J. Food Prot. 58:993-997.

19. Sammarco, M. L., G. Ripabelli, A. Ruberto, G. Iannitto, and G. M. Grasso. 1997. Prevalence of salmonellae, listeriae, and yersinae in the slaughterhouse environment and on work surfaces, equipment, and workers. J. Food Prot. 60:367-371.

20. Silliker, J. H., and D. A. Gabis. 1975. A cellulose sponge sampling technique for surfaces. J. Food Prot. 38:504.

21. Skovgaard, N., and C. A. Morgen. 1988. Detection of Listeria spp. in faeces from animals, in feeds, and in raw foods of animal origin. Int. J. Food Microbiol. 6:229-242.

22. Slade, P. J. 1992. Monitoring Listeria in the food production environment. I. Detection of Listeria in processing plants and isolation methodology. Food Res. Int. 25:45-56.

23. Tenover, F. C., R. D. Arbeit, R. V. Goering, P. A. Mickelsen, B. E. Murray, D. H. Persing, and B. Swaminathan. 1995. Interpreting chromosomal DNA restriction patterns produced by pulsed-field gel electrophoresis: criteria for bacterial strain typing. J. Clin. Microbiol. 33: 2233-2239.

24. Unnerstad, H., E. Bannerman, J. Bille, M. L. Danielsson-Tham, E. Waak, and W. Tham. 1996. Prolonged contamination of a dairy with Listeria monocytogenes. Neth. Milk Dairy J. 50:493-499.

25. Wagner, M., A. Lehner, and E. Brandl. 1998. Comparison of three methods for molecular differentiation of foodborne and clinical Listeria strains: single strand conformation polymorphisms, random amplified polymorphic DNA polymorphisms and pulsed-field gel electrophoresis. Arch. Lebensmittelhyg. 49:57-61.

26. Warburton, D. W., J. M. Farber, A. Armstrong, R. Caldeira, N. P. Tiwari, T. Babiuk, P. Lacasse, and S. Read. 1991. A Canadian comparative study of modified versions of the "FDA" and "USDA" methods for the detection of Listeria monocytogenes. J. Food Prot. 54:669-676. 